\title{
MULTIPLE OPIAL-TYPE INEQUALITIES FOR GENERAL KERNELS WITH APPLICATIONS
}

\section{SAJID IQBAL, Josip PEČARIĆ AND MUHAMMAD SAMRAIZ}

Abstract. The main purpose of this paper is to give the general multiple Opial-type inequalities for general kernels. We consider the monotocity and boundedness of the weight functions to prove new inequalities. As applications of our general results we establish new inequalities for Widder's derivatives and linear differential operator. Results from [11] are obtain by applying the Canavati fractional derivatives to our main results.

Mathematics subject classification (2010): 26D15, 26D10, 26A33.

Keywords and phrases: Opial's inequality, kernel, fractional derivative, Widder's derivatives, linear differential operators, Green's function.

\section{REFERENCES}

[1] R. P. AgARWAL, Sharp Opial-type inequalities involving r-derivatives and their applications, Tohoku Math. J. 47 (1995), 567-593.

[2] R. P. Agarwal, P. Y. H. PAng, Opial Inequalities with Applications in Differential and Difference Equations, Kluwer Acad. Publ., Dordrecht, 1995.

[3] R. P. Agarwal, P. Y. H. PAng, Sharp Opial-type inequalities involving higher order derivatives of two functions, Math. Nachr. 174 (1995), 5-20.

[4] R. P. Agarwal, P. Y. H. PAng, Opial Inequalities with Applications in Differential and Difference Equations, Kluwer Acad. Publ., Dordrecht, 1995.

[5] H. Alzer, On some inequalities of Opial-type, Arch. Math. 63 (1994), 431-436.

[6] H. AlzER, An Opial-type inequality involving higher-order derivativess ot two functions, Appl. Math. Lett. 10, 4 (1997), 123-128.

[7] G. A. Anastassiou, J. PeČARIĆ, General Opial inequalites for linear differential opeators, J. Math. Anal. Appl. 239 (1999), 402-418.

[8] G. A. Anastassiou, Advanced inequalities. Vol. 11, World Scientific, 2011.

[9] G. A. Anastassiou, Opial type inequalities for linear differential operators, Math. Inequal. Appl. 1 (1998), 193-200.

[10] M. ANDRIĆ, J. PEČARIĆ, I. PERIĆ, Improvements of composition rule for Canavati fractional derivative and applications to Opial-type inequalities, Dynam. Systems Appl. 20 (2011), 383-394.

[11] M. Andrić, J. PeČArić, I. Perić, General multiple Opial-type inqualities for the Canavati fractional derivatives, Ann. Funct. Anal. 4 (2013), 149-162.

[12] W. S. Cheung, Z. DANDAN, J. PeČARIĆ, Opial-type inequalities for differential operators, Nonlinear Analysis 66 (2007), 2028-2039.

[13] D. S. Mitrinović, J. PeČArić And A. M. FinK, Classical and new inequalities in analysis, Kluwer Academic Publisher, 1993.

[14] Z. Opial, Sur une inegalite, Ann. Polon. Math. 8 (1960), 29-32.

[15] B. G. Pachpatte, On Opial-type inéqualitiés, J. Math. Anal. Appl. 120 (1986), 547-556. 
[16] D. V. WIDDER, A Generalization of Taylor's Series, Transactions of AMS, 30, 1 (1928), 126-154. 